\title{
Tradução, adaptação cultural e validação do Frail Non-Disabled Questionnaire para o contexto brasileiro
}

\author{
Translation, adaptation and validation of Frail Non-Disabled Questionnaire to the brazilian \\ context
}

Rafaela Brochine Lanzotti 1 (D) Vanessa Almeida Maia Damasceno' $\mathbb{I D}^{\mathrm{D}}$ Fabiana de Souza Orlandi? ${ }^{\mathbb{D}}$

\section{Resumo}

Objetivo: Traduzir, adaptar e validar o Frail Non-Disabled Questionnaire (FiND) para o contexto brasileiro. Método: Foram seguidas as etapas preconizadas para instrumentos da área da saúde: tradução inicial, síntese das traduções, retrotradução, revisão por um comitê de especialistas, pré-teste e avaliação das propriedades psicométricas da escala. Todos os preceitos éticos foram seguidos. Resultados: A tradução e a retrotradução foram realizadas por dois tradutores independentes e qualificados. A revisão por especialistas demonstrou sua validade de conteúdo. No pré-teste, o FiND foi de fácil compreensão e aplicação. Na avaliação das propriedades psicométricas, o instrumento demonstrou valores satisfatórios de confiabilidade e reprodutibilidade. A validade de critério concorrente foi verificada, encontrando correlação positiva com significância estatística entre o escore do FiND e do Fenótipo de Fragilidade de Fried, e valores satisfatórios de sensibilidade, especificidade, valores preditivos positivo e negativo, e acurácia. A validade de construto convergente foi analisada, indicando correlação positiva estatisticamente significante entre o escore do FiND e os sintomas depressivos, e correlações negativas com o estado nutricional, cognitivo, e com os domínios do componente físico da qualidade de vida. A validade discriminante foi analisada por meio da comparação das médias do FiND entre os grupos: robusto, não frágil e frágil, com e sem sintomas depressivos, com melhor e com pior percepção de qualidade de vida, e com estado nutricional adequado, sob risco de desnutrição e com estado de desnutrição, as quais mostraram-se estatisticamente significantes. Conclusão: O FiND encontra-se traduzido, adaptado e validado para o contexto brasileiro.

\footnotetext{
Universidade Federal de São Carlos, Programa de Pós-Graduação em Enfermagem. São Paulo, SP, Brasil.

Financiamento da pesquisa: Coordenação de Aperfeiçoamento de Pessoal de Nível Superior - Brasil (CAPES) - Código de Financiamento 001.

Os autores declaram não haver conflito na concepção deste trabalho.

Palavras-chave: Tradução. Estudos de Validação. Fragilidade. Idoso. Questionário. 


\section{Abstract}

Objective: To translate, adapt and validate the Frail Non-Disabled Questionnaire (FiND) for the Brazilian context. Method: The steps recommended by the international literature for health instruments were followed: initial translation, synthesis of translations, backtranslation, review by a committee of experts, pre-test, and assessment of the scale's psychometric properties. All ethical precepts were followed. Results: The translation and back-translation were performed by two independent and qualified translators. The expert review demonstrated its content validity. In the pre-test, FiND was easy to understand and apply. In the assessment of psychometric properties, the instrument demonstrated good values of reliability and reproducibility. Concurrent criterion validity was verified, finding a positive correlation with statistical significance between the FiND score and Fried's Fragility Phenotype and good values of sensitivity, specificity, positive and negative predictive values, and accuracy. The convergent construct validity was analyzed, indicating a positive correlation between the FiND score and depressive symptoms, and negative correlations with nutritional and cognitive status, and with the domains of the physical component of quality of life, with statistical significance. Discriminant validity was analyzed by comparing FiND means between the robust, non-frail, and fragile groups, with and without depressive symptoms, with a better and worse perception of quality of life, and with adequate nutritional status, at risk of malnutrition and with a state of malnutrition, which proved to be statistically significant. Conclusion: FiND has been translated, adapted, and validated for the Brazilian context.

\section{INTRODUÇÃO}

O envelhecimento populacional é reconhecido como um processo dinâmico, progressivo e irreversível ${ }^{1}$. Em decorrência do rápido declínio nas funções físicas e mentais das pessoas idosas, verificase um aumento da sobrecarga nos sistemas de saúde e assistência social em todo o mundo devendo-se priorizar ações preventivas da deterioração funcional da população idosa ${ }^{2}$. Nesse contexto, a avaliação multidimensional com foco nas síndromes geriátricas torna-se necessária, atentando-se para a identificação dessas e para o seu tratamento, produzindo melhores resultados para a assistência ao idoso, visto que são as principais responsáveis pela perda de autonomia e independência do idoso ${ }^{3}$.

A fragilidade é definida como uma síndrome, a qual é caracterizada por uma diminuição energética e está relacionada a mudanças fisiológicas dos sistemas: musculoesquelético, neuroendócrino e imunológico. Desencadeando, portanto, uma redução da massa muscular, distúrbios do apetite e uma inflação crônica ${ }^{4}$. A identificação da fragilidade é fundamental para a implementação de intervenções preventivas multidimensionais, as quais favorecerão melhor qualidade de vida ${ }^{5}$.
Diversos instrumentos foram desenvolvidos ao longo dos anos para identificar a fragilidade em pacientes idosos ${ }^{6}$. Entretanto, as ferramentas de triagem disponíveis na literatura apresentam duas principais limitações: poucas são válidas para a autoadministração e poucas permitem diferenciar a fragilidade da incapacidade - termo amplo para indicar deficiências, limitações no desempenho de atividades e restrições na participação social, associadas aos estados de saúde ${ }^{5}$.

Nesse contexto, Cesari et al. ${ }^{4}$ elaboraram o instrumento FrailNon-Disabled Questionnaire (FiND), na França, o qual é autoadministrado, composto por cinco itens e segue a principal construção multidimensional do fenótipo de fragilidade, amplamente adotado proposto por Fried et al. ${ }^{5}$.

Não somente, o instrumento ainda aborda uma seção específica para excluir a presença de deficiência de mobilidade, a qual é considerada a fase inicial do processo de incapacitação, e pode representar uma oportunidade para difundir a conscientização sobre a fragilidade e a incapacidade em pessoas idosas 5 .

Frente ao exposto, a disponibilização do FiND para amplo uso no Brasil é relevante e essencial.
Keywords: Translating.
Validation Study. Frailty. Elderly. Questionnaire. 
Ademais, até o momento, não foram encontrados instrumentos que permitem a diferenciação entre idosos incapazes e frágeis na literatura brasileira. Desse modo, pretende-se com o presente trabalho realizar a tradução, a adaptação cultural e a validação do Frail Non-Disabled Questionnaire (FiND) para o contexto brasileiro.

\section{MÉTODO}

Trata-se de um estudo metodológico de tradução, adaptação cultural e validação do instrumento Frail Non-Disabled Questionnaire (FiND) ${ }^{5}$ para o contexto brasileiro. Para o processo de tradução, adaptação e validação do FiND foram seguidas sequencialmente as etapas preconizadas pela literatura?

Tal processo contou com sete fases, sendo elas, respectivamente: tradução inicial; síntese das traduções; retrotradução; revisões por um comitê de juízes; pré-teste; apresentação e avaliação dos relatórios do processo de adaptação cultural; avaliação das propriedades psicométricas do instrumento, após sua tradução e adaptação cultural ${ }^{7}$.

Inicialmente, o instrumento FiND foi submetido à tradução do inglês americano para o português brasileiro por dois tradutores qualificados e bilíngues, de maneira independente. Após, os pesquisadores e os tradutores definiram uma versão consensual em português brasileiro do FiND, avaliando as diferenças existentes nas traduções, considerando a versão original do instrumento.

Seguindo o referencial teórico adotado, a versão consensual em português brasileiro do FiND foi revertida para o inglês americano por outro tradutor qualificado, com o inglês americano como língua materna, e com experiência na tradução de textos na área da saúde. $O$ tradutor não conhecia os objetivos do presente estudo ou a versão original do questionário utilizado.

A versão retrotraduzida apresentou semelhanças com o instrumento original em inglês americano. Desse modo, a versão consensual em português brasileiro do FiND e a versão retrotraduzida foram avaliadas pelo comitê de juízes.
O comitê de especialistas, foi composto por cinco juízes. A seleção baseou-se nos seguintes requisitos: fluência na língua inglesa, formação na área de saúde, experiência na temática de fragilidade em idosos, bem como conhecimento da metodologia da pesquisa, ou seja, possuir experiência com tradução e adaptação de instrumentos. Ainda, vale ressaltar que a seleção dos membros participantes do comitê de especialistas ocorreu por meio da consulta à plataforma lattes (www.lattes.cnpq.br). Os mesmos, avaliaram a equivalência semântica, idiomática, experimental e conceitual da versão consensual do FiND, produzindo sua versão pré-final.

Na sequência foi realizado o pré-teste, para essa etapa foram selecionados 46 idosos da comunidade que eram atendidos pelas Unidades de Saúde da Família (USF) de uma cidade do interior do estado de São Paulo. Nesta etapa de avaliação do instrumento FiND os participantes analisaram a clareza e a compreensão de todos os itens que compõem o FiND. Uma vez realizado o pré-teste e estabelecida a versão final do FiND, deu-se início à última etapa referente à avaliação das propriedades psicométricas do instrumento, incluindo a avaliação da confiabilidade e validade do instrumento.

A amostra para a etapa de avaliação das propriedades psicométricas do instrumento foi composta por 234 idosos atendidos pelas USF de uma cidade do interior do estado de São Paulo. Os critérios de inclusão foram: idade igual ou superior a 60 anos e serem atendidos pelas USF do município. Os critérios de exclusão foram: indivíduos que possuíam grave deficit de visão ou audição referido; alteração cognitiva rastreada pelo Exame Cognitivo de Addenbrooke - Versão Revisada (ACE-R) ${ }^{8}$ e pessoas com limitações funcionais devido a doenças agudas ou crônicas que impediam o participante de realizar parte do Fenótipo de Fragilidade de Fried ${ }^{4}$ (teste de preensão palmar e teste de caminhada).

Os instrumentos utilizados na avaliação das propriedades psicométricas, para a análise da validade de construto, foram aplicados, além do FiND ${ }^{5}$, o Fenótipo de Fragilidade de Fried $^{4}$, o ACE-R ${ }^{8}$, a Escala de Depressão Geriátrica - versão reduzida $\left(\right.$ GDS-15) ${ }^{9}$, a Mini Avaliação Nutricional (MAN) ${ }^{10}$ e o Short Form - 36 (SF-36) ${ }^{11}$. Os instrumentos 
foram aplicados por meio de entrevista individual, conduzida por três discentes de pós-graduação, devidamente treinadas.

O FiND foi elaborado por Cesari e et al. ${ }^{5}$ na língua inglesa na França em 2014, o mesmo é composto por cinco itens, sendo os dois primeiros (A e B) destinados a identificar pacientes com incapacidades, enquanto os três últimos (C, D e E) são destinados à avaliação dos sinais, sintomas ou condições geralmente consideradas como componentes da síndrome de fragilidade ${ }^{5}$. Sua pontuação total varia de zero a 5 pontos. Caso o paciente idoso pontue nos itens $\mathrm{A}$ ou $B$, é considerado incapaz. Se não pontuar nos itens A e B, mas pontuar no $\mathrm{C}, \mathrm{D}$ ou E, é considerado frágil. Por fim, se não pontuar em nenhum item, o idoso é considerado robusto/não frágil ${ }^{5}$.

O Fenótipo de Fragilidade de Fried foi elaborado por Fried et al. ${ }^{4}$, como uma avaliação para a síndrome da fragilidade. Para tal, é composto por cinco critérios que avaliam a condição de fragilidade. O ACE-R é um instrumento útil no diagnóstico de demência em fases iniciais. De modo a facilitar sua aplicação e adicionar novas questões de linguagens, no Brasil, a versão revisada foi traduzida e validada por Carvalho e Caramelli em $2007^{8}$, sendo composta por questões que avaliam cinco domínios cognitivos.

O GDS-15 é frequentemente utilizado para a detecção de sintomas depressivos em pacientes idosos. No Brasil, a versão reduzida de 15 questões (GDS15) foi traduzida e validada por Almeida e Almeida em $1999^{\circ}$. A MAN foi elaborada por Vellas et al. ${ }^{10}$ em 1999, e refere-se a uma ferramenta que fornece um método simples e rápido para a identificação de pacientes idosos que apresentam risco de desnutrição ou que já estejam desnutridos. Já o SF-36 refere-se a um instrumento genérico de avaliação da qualidade de vida, de fácil administração e compreensão, o mesmo foi traduzido e validado para o contexto brasileiro por Ciconelli et al. ${ }^{11}$ em 1999.

Para a análise dos dados, foram realizadas análises descritivas e a verificação da normalidade dos dados, por meio do Teste de Kolmogorov-Smirnov.

Para verificação da validade de conteúdo do FiND utilizou-se o Índice de Validade de Conteúdo (IVC) para avaliação das respostas dos especialistas referente a cada item presente no FiND. O instrumento de avaliação dos juízes foi composto por uma escala de resposta do tipo Likert de 1 a 4 pontos, sendo que para cada item da escala o especialista poderia considerar o item: $1=$ não equivalente; $2=$ pouco equivalente; $3=$ equivalente; $4=$ muito equivalente. Para a interpretação do IVC foi adotado o valor recomendável de 0,80 ou mais. O escore do IVC foi calculado por meio da soma de concordância dos itens que receberam pontuações de " 3 " e " 4 " pelos especialistas, dividido pelo número total de respostas.

Para a análise das propriedades psicométricas do FiND, verificou-se: a consistência interna do instrumento, por meio do Kuder-Richardson 20 $(\mathrm{KR}-20)^{12}$; a estabilidade do escore do FiND, por meio do Coeficiente de Correlação Intraclasse ${ }^{13}$; a validade de critério concorrente do FiND, por meio do Coeficiente de Correlação de Spearman, relacionando o seu escore obtido com o Fenótipo de Fragilidade de Fried ${ }^{4}$, em que a magnitude das correlações foi classificada conforme proposição de Levin e Fox ${ }^{14}$. Não somente, para verificar a validade de critério concorrente do instrumento realizouse ainda a análise dos valores de sensibilidade, especificidade, acurácia e valores preditivos positivos e negativos, com respectivos intervalos de $95 \%$ de confiança, além da relação linear quanti-qualitativa entre a área sob a curva ROC (AUC) ${ }^{15}$; a validade de construto convergente do instrumento FiND, por meio do Coeficiente de Correlação de Spearman, relacionando seu escore obtido com os escores dos domínios do componente físico do SF-36, do ACE-R, da MAN e do GDS- $15^{16}$.

Além disso, realizou-se os testes de KruskalWallis e de Mann Whitney para a verificação da validade discriminante do instrumento do FiND, segundo o nível de fragilidade avaliado pelo fenótipo de fragilidade de Fried (robusto, não frágil e frágil); o nível de sintomas depressivos (sem sintomas depressivos, com sintomas depressivos leves e severos); a percepção de qualidade de vida (melhor e pior); o estado nutricional (estado de desnutrição, sob risco de desnutrição e estado nutricional adequado); e cognitivo (com indícios de alteração cognitiva e sem indícios). 
O nível de significância adotado foi de 5\%, $(\mathrm{p}$-valor $\leq 0,05)$. Esta pesquisa foi aprovada pelo Comitê de Ética com Seres Humanos da Universidade Federal de São Carlos (Parecer Número 1891428) foram respeitados todos os preceitos éticos dispostos nas Resoluções 466/12 e 510/2016 do Conselho Nacional de Saúde.

\section{RESULTADOS}

Seguindo as etapas desenvolvidas na pesquisa, obteve-se duas versões traduzidas do FiND, além de uma versão consensual e uma versão retrotraduzida para o idioma original.

A versão retrotraduzida apresentou semelhanças com o instrumento original em inglês americano. Desse modo, a versão consensual em português brasileiro do FiND e a versão retrotraduzida foram avaliadas pelo comitê de juízes.

Após a avaliação do comitê dos juízes verificouse que dos 21 itens que compõem o FiND, 14 apresentaram valores de IVC $=1$, sendo considerados equivalentes e mantidos na versão pré-final do instrumento. Os demais itens foram reanalisados e modificados pelas pesquisadoras, conforme a sugestão dos especialistas. Após as modificações, a versão pré-final foi reenviada e aprovada por todos os juízes. Subsequentemente, obteve-se a versão pré-final do instrumento e o mesmo foi testado com 46 idosos da comunidade que atendiam aos critérios de elegibilidade. Nessa etapa, a versão aplicada não foi alterada, sendo julgada como clara, rápida e de fácil compreensão. Assim, não foram sugeridas modificações.

Por fim, na última etapa de avaliação das propriedades psicométricas dos instrumentos, o estudo contou com 234 idosos. Foi observado predominância de respondentes do sexo feminino
(67,95\%), de etnia branca $(73,93 \%)$ e casados $(59,40 \%)$. Com relação à escolaridade dos participantes, observou-se predomínio de idosos com 1 a 4 anos de estudo (51,30\%), seguido por idosos com mais de 9 anos de estudo (18,80\%), com 5 a 8 anos de escolaridade $(17,94 \%)$ e com menor predomínio, observou-se os idosos analfabetos $(11,96 \%)$. Verificouse ainda que houve a predominância de idosos católicos (70,51\%), praticantes de alguma religião $(64,22 \%)$, aposentados ou pensionistas $(79,49 \%)$, que viviam em residências próprias $(91,45 \%)$, que utilizavam pelo menos um medicamento de maneira contínua $(88,89)$, e que não tinham sofrido quedas nos últimos 12 meses $(64,53)$.

Com relação à confiabilidade do instrumento, o valor encontrado para o coeficiente KR-20 foi de 0,642 ; entretanto, verificou-se que o item C (3) apresentava menor consistência e quando excluído, o valor encontrado era de 0,705 , considerado satisfatório. No entanto, esta questão é de suma importância para o instrumento, pois se trata da perda de peso, portanto, optou-se por deixá-la na escala. Observou-se também satisfatória estabilidade, uma vez que o valor encontrado para o teste-reteste foi de ICC $=0,841$ (IC 95\% 0,718; 0,910).

O FiND apresentou satisfatória validade de critério concorrente ao ser correlacionado ao instrumento considerado padrão ouro para a avaliação da fragilidade física. Observou-se a existência de correlação positiva, de forte magnitude e com significância estatística entre o FiND ${ }^{5}$ e o Fenótipo de Fragilidade de Fried ${ }^{4}(r=0,603 ; p<0,001)$.

Não somente, verificou-se satisfatória validade de critério concorrente do instrumento FiND por meio da análise da Curva ROC. A Curva ROC traçada para a escala encontra-se apresentada na figura 1 , a área sob a Curva ROC traçada para a pontuação obtida no FiND atingiu o valor satisfatório de 0,855 (IC 95\% [0,793;0,917]; $p<0,001$ ). 


\section{Curva ROC}

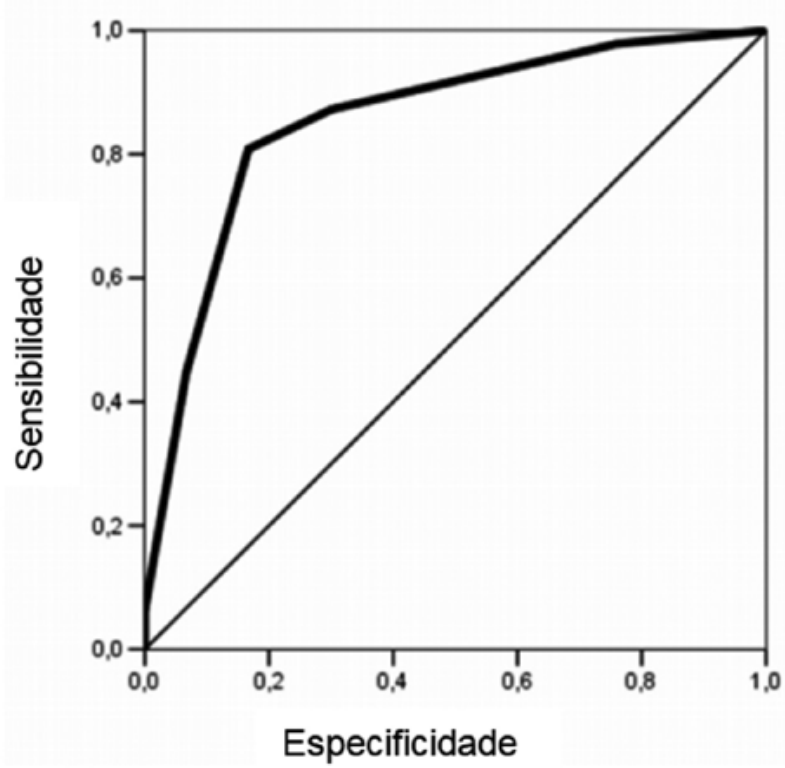

Figura 1. Curva ROC para FiND, usando o Fenótipo de Fragilidade de Fried como padrão ouro. São Carlos, 2018.

A análise dos valores permitiu constatar que o ponto de corte do FiND ideal para o rastreio de fragilidade é de 2,5 , resultante em sensibilidade de $80,85 \%$ [IC 95\% (50,88; 97,06)] e especificidade de $83,33 \%$ [IC 95\% $(75,71 ; 94,51)]$. Os valores preditivos positivo e negativo para esse ponto de corte foram de 55,88\% [IC 95\% (33,45; 80,57)] e 94,34\% [IC 95\% $(85,67 ; 99,33)]$, respectivamente. O valor de acurácia do instrumento foi de 86,96\% [IC 95\% $(76,18 ; 93,50)]$.

Verifica-se na Tabela 1, que o FiND apresentou satisfatória validade de critério convergente ao ser correlacionado com os escores dos instrumentos
ACE-R, GDS-15, MAN, e dos domínios do componente físico do SF-36 (Capacidade Funcional, Aspectos Físicos, Dor e Estado Geral de Saúde).

Verifica-se na Tabela 2, que o FiND apresentou satisfatória validade de construto discriminante, uma vez que o instrumento conseguiu diferenciar os grupos, segundo o nível de fragilidade (avaliado pelo fenótipo de fragilidade de Fried), a presença de sintomas depressivos (avaliado pelo GDS-15), a percepção de qualidade de vida (avaliada pelo SF-36), o estado nutricional (avaliado pela MAN) e cognitivo (avaliado pelo ACE-R).

Tabela 1. Análise da validade de construto convergente do instrumento FiND por meio da análise do Coeficiente de Correlação de Spearman com o GDS-15, ACE-R, MAN e os domínios do componente físico do SF-36. São Carlos, 2017-2018.

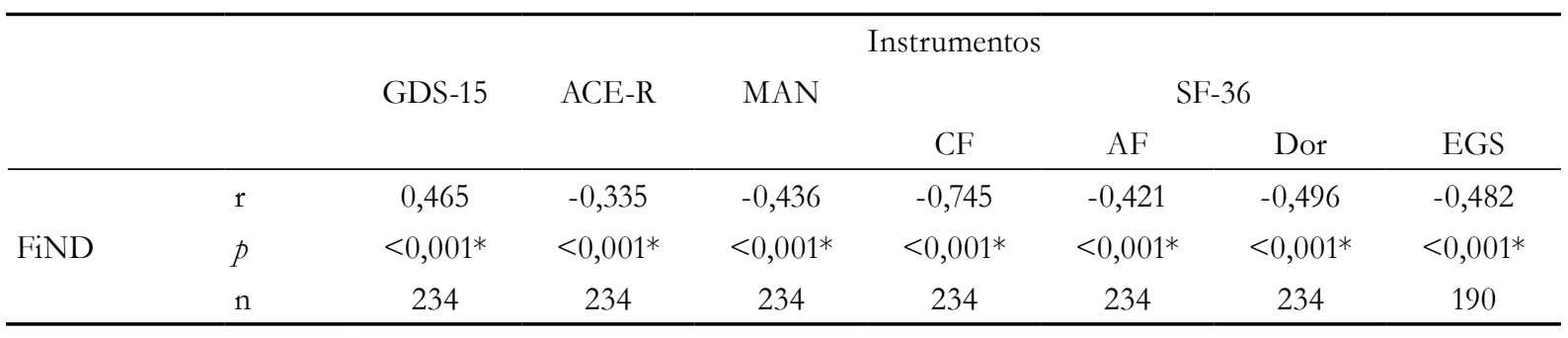

r: Coeficiente de correlação de Spearman; $p$ : Valor do p; n: Tamanho amostral; * Estatisticamente significante; CF: Capacidade Funcional; AF: Aspectos Físicos; EGS: Estado Geral de Saúde. 
Tabela 2. Análise da validade de construto discriminante da versão brasileira do FiND. São Carlos, São Paulo, Brasil, 2017-2018.

\begin{tabular}{|c|c|c|c|}
\hline \multirow{2}{*}{ Instrumento / Categorias } & \multicolumn{2}{|c|}{ FiND } & \multirow[t]{2}{*}{$p$ value } \\
\hline & $\mathrm{N}$ & Média \pm DP* & \\
\hline \multicolumn{4}{|l|}{ Fenótipo de Fragilidade de Fried } \\
\hline Robusto & 49 & $0,59 \pm 0,73$ & $<0,001^{* *, a}$ \\
\hline Pré-Frágil & 131 & $1,56 \pm 1,15$ & \\
\hline Frágil & 48 & $3,19 \pm 1,14$ & \\
\hline \multicolumn{4}{|l|}{ MAN } \\
\hline Estado de Desnutrição & 6 & $4,33 \pm 0,82$ & $<0,001^{* *, \mathrm{~b}}$ \\
\hline Sob Risco de Desnutrição & 28 & $2,93 \pm 1,33$ & \\
\hline Estado Nutricional Normal & 200 & $1,44 \pm 1,21$ & \\
\hline \multicolumn{4}{|l|}{ ACE-R } \\
\hline Com Indício de Alteração Cognitiva & 127 & $1,97 \pm 1,47$ & $0,002^{* * *}$ \\
\hline Sem Indício de Alteração Cognitiva & 107 & $1,36 \pm 1,18$ & \\
\hline \multicolumn{4}{|l|}{ GDS-15 } \\
\hline Sem Sintomas Depressivos & 157 & $1,29 \pm 1,18$ & \\
\hline Sintomas Depressivos Leves & 60 & $2,3 \pm 1,41$ & $<0,001^{* *, c}$ \\
\hline Sintomas Depressivos Severos & 16 & $3,25 \pm 1,12$ & \\
\hline \multicolumn{4}{|l|}{ SF-36 } \\
\hline \multicolumn{4}{|l|}{ Capacidade Funcional } \\
\hline Pior qualidade de vida & 119 & $2,51 \pm 1,30$ & $<0,001^{* * *}$ \\
\hline Melhor qualidade de vida & 110 & $0,75 \pm 0,68$ & \\
\hline \multicolumn{4}{|l|}{ Avaliação Funcional } \\
\hline Pior qualidade de vida & 60 & $2,62 \pm 1,43$ & $<0,001^{* * *}$ \\
\hline Melhor qualidade de vida & 169 & $1,33 \pm 1,18$ & \\
\hline \multicolumn{4}{|l|}{ Dor } \\
\hline Melhor qualidade de vida & 114 & $2,27 \pm 1,39$ & $<0,001^{* * *}$ \\
\hline Pior qualidade de vida & 115 & $1,06 \pm 1,05$ & \\
\hline \multicolumn{4}{|l|}{ Estado geral de Saúde } \\
\hline Pior qualidade de vida & 118 & $2,24 \pm 1,42$ & $<0,001^{* * *}$ \\
\hline Melhor qualidade de vida & 111 & $1,05 \pm 1,02$ & \\
\hline
\end{tabular}

*DP=Desvio-Padrão; **Teste de Kruskal-Wallis; ${ }^{* * *}$ Teste de Mann Whitney; ${ }^{2}$ Diferenças estatísticas entre os grupos robusto e pré-fragil, robusto e frágil, e frágil e pré-fragil; biferenças estatísticas entre os grupos sob estado de desnutrição e estado nutricional normal, e sob risco de desnutrição e estado nutricional normal; ' Diferenças estatísticas entre os grupos com ausência de sintomas depressivos e com sintomas depressivos leves, e com ausência de sintomas depressivos e com sintomas depressivos severos;

\section{DISCUSSÃO}

O presente estudo traduziu, adaptou e validou o FiND para o contexto brasileiro, propondo-se a preencher uma lacuna existente na área de atenção à saúde do idoso no Brasil. Para os processos de tradução e adaptação, foram seguidas as etapas preconizadas pela literatura, contribuindo significativamente para a qualidade do resultado obtido, indicando que a versão em português do instrumento é linguisticamente fiel ao questionário em sua língua original (inglês), sendo a sua adequação confirmada pela avaliação de um comitê composto por especialistas.

Vários instrumentos são produzidos em um idioma e, posteriormente, traduzidos para outros, 
e a etapa de validação por especialistas é muito importante nesses processos de tradução e adaptação cultural e linguística das escalas e questionários ${ }^{17}$. Segundo Alexandre e Coluci ${ }^{18}$, a tradução e adaptação de um instrumento é um processo complexo devido à existência de diferenças culturais e de linguagem entre os países envolvidos. Deste modo, a seleção inadequada de instrumentos de medição de baixa qualidade metodológica pode ser considerada um viés nas conclusões de estudos, sendo altamente justificada a padronização na tradução e adaptação de instrumentos ${ }^{19}$.

A amostra estudada foi descrita quanto às características sociodemográficas. Houve a predominância de idosos do sexo feminino, com baixa escolaridade (1 a 4 anos), brancos e casados , corroborando com diversos outros estudos disponíveis na literatura nacional e internacional ${ }^{20-22}$. Ainda é possível verificar que a literatura nos evidência que que pertencer ao gênero feminino e ter idade avançada podem ser preditores de fragilidade para a fragilidade ${ }^{21,23,24}$.

Com relação às propriedades psicométricas do instrumento, a homogeneidade e a reprodutibilidade do FiND foram verificadas por meio da análise da consistência interna do instrumento e de seu testereteste, sendo considerados satisfatórios os resultados obtidos. Diversos instrumentos disponíveis na literatura para a avaliação da fragilidade apresentaram satisfatória homogeneidade e reprodutibilidade, sendo utilizados em estudos de grandes escalas. Como exemplo, cita-se os instrumentos Frailty Index, PRISMA-7, CHS Index e FRAIL 4,24-26.

Não somente, observou-se satisfatória validade de critério concorrente do instrumento FiND, visto que este se correlacionou de maneira positiva e forte com o instrumento considerado padrão ouro para a avaliação da fragilidade física, e apresentou resultados satisfatórios por meio da análise da Curva ROC, demonstrando satisfatória sensibilidade, especificidade, valores preditivos positivo, negativo e acurácia.

No estudo da versão original do instrumento, os dados sobre a confiabilidade da escala não foram informados. Com relação à sua validade, encontraram-se apenas dados sobre sua acurácia, por meio de sua especificidade e capacidade de identificação de pacientes frágeis não incapazes, corroborando com os dados encontrados no presente estudo. Nesse contexto, os autores concluíram que o instrumento FiND apresentou boa capacidade para identificar corretamente idosos frágeis sem incapacidades vivendo na comunidade ${ }^{4}$.

Ainda com relação à acurácia do instrumento FiND, Mirabelli et al. ${ }^{27}$ utilizaram o instrumento para avaliar a fragilidade na prática clínica com pacientes com doenças vasculares, e observaram uma boa sensibilidade, porém, uma baixa especificidade. Os dados sobre a confiabilidade do instrumento não foram informados.

No presente estudo, observou-se ainda satisfatória validade de construto discriminante, uma vez que o instrumento FiND foi capaz de discriminar os grupos de idosos segundo o nível de fragilidade, presença de sintomas depressivos, percepção de qualidade de vida, estado nutricional e cognitivo, corroborando com diversos estudos que investigam a relação entre as variáveis estudadas ${ }^{28-32}$.

Rossetti et al. ${ }^{28}$ investigaram a relação entre a fragilidade e os sintomas depressivos e sobrecarga de idosos cuidadores em contexto de alta vulnerabilidade social, e observaram correlação positiva e de moderada magnitude entre a fragilidade e os sintomas depressivos, concluindo que, à medida que os níveis de fragilidade aumentavam, os sintomas depressivos se tornavam mais prevalentes na população estudada. Não somente, Ribeiro et al..$^{29}$ exploraram a relação entre sintomas depressivos e fragilidade em 91 idosos centenários de duas regiões diferentes de Portugal e observaram que os centenários que foram classificados como frágeis apresentaram maiores riscos de depressão quando comparados com os centenários pré-frágeis, concluindo que a depressão é uma condição frequente em idosos frágeis.

Referente à relação da fragilidade e da qualidade de vida, Jesus et al. ${ }^{30}$ avaliaram o nível de fragilidade e sua relação com a percepção de qualidade de vida de idosos cadastrados em Centros de Referências de 
Assistência Social de um município do interior do estado de São Paulo, Brasil e observaram correlações negativas e de fraca à moderada magnitude entre a fragilidade e a qualidade de vida, indicando que os idosos mais frágeis apresentaram pior qualidade de vida. Em seu estudo de revisão sistemática, Kojima et al. ${ }^{31}$ concluíram que pacientes idosos classificados como frágeis ou pré-frágeis apresentaram qualidade de vida física e mental significantemente menor do que aqueles classificados como não frágeis.

Com o intuito investigar a relação entre a fragilidade e os parâmetros nutricionais relatados por adultos residentes dos Estados Unidos e observaram a relação entre os parâmetros nutricionais e a fragilidade, os quais contribuem com o aumento no risco de morte ${ }^{32}$.

De modo a analisar a relação entre a cognição e a fragilidade em pessoas idosas, Brigola et al. ${ }^{24}$ realizaram uma revisão sistemática da literatura sobre o assunto, analisando 19 estudos. Como resultado, todos os estudos estabeleceram uma relação entre a cognição e a fragilidade, em que componentes da fragilidade e os domínios cognitivos apresentaramse relacionados. Ainda, Hao et al..$^{32}$ investigaram o impacto da fragilidade e do comprometimento cognitivo em 705 idosos da comunidade chinesa e concluíram que os idosos que apresentavam as duas condições concomitantemente foram associadas com o aumento no risco de morte, sendo a fragilidade e o comprometimento cognitivo fatores de risco para a morte em pessoas idosas.

Frente ao exposto, confirmam-se as evidências de confiabilidade e validade da versão brasileira do FiND, sendo esse instrumento disponibilizado para amplo uso no Brasil, tendo em vista que a identificação e o rastreio precoce da fragilidade, realizados por meio de ferramentas simples por profissionais da área da saúde, cuidadores e familiares tornam-se fundamentais para a implementação de ações e favorecem a melhoria da qualidade de vida da população em processo de fragilização.

No presente estudo, como limitações, destaca-se o recorte transversal, o qual não permitiu a verificação da responsividade do instrumento FiND, não sendo possível, portanto, verificar sua sensibilidade para a detecção de mudanças. Não somente, não foi possível encontrar estudos de tradução, adaptação e validação do instrumento FiND para outros contextos, o que dificultou a comparação dos resultados obtidos com outros estudos, limitando a discussão mediante os resultados apresentados. Além disso, vale citar que o FiND foi elaborado com base no fenótipo de fragilidade de Fried e adotou-se o mesmo como critério para testar a validade concorrente, por fim, cita-se ainda a não adoção de um instrumento que avaliasse especificamente a incapacidade, como foi feito no estudo original da elaboração do instrumento.

\section{CONCLUSÃO}

Com base nos objetivos propostos e resultados obtidos, pode-se concluir que o instrumento FiND encontra-se traduzido, adaptado e validado para o contexto brasileiro (material suplementar). $\mathrm{O}$ mesmo demonstrou satisfatórias propriedades psicométricas (confiabilidade, validade de critério concorrente, de construto convergente e discriminante).

Espera-se que este estudo auxilie, por meio da disponibilização da versão brasileira do FiND, no rastreio de fragilidade da população idosa brasileira, possibilitando assim a implementação de ações pelos profissionais de saúde, com intuito de reverter ou mesmo de prevenir a referida síndrome. Recomendase também a realização de novos estudos, a fim de ampliar e confirmar as propriedades psicométricas do FiND versão brasileira em diferentes populações e contextos.

Editado por: Maria Helena Rodrigues Galvão 


\section{REFERÊNCIAS}

1. Wallace LMK, Theou O, Pena F, Rockwood K, Andrew MK. Social vulnerability as a predictor of mortality and disability: cross-country differences in the survey of health, aging, and retirement in Europe (SHARE). Aging Clin Exp Res. 2015;27(3):1-10. Disponível em: https://doi. org/10.1007 / s40520-014-0271-6 .

2. Morley JE, Little MO, Berg-Weger M. Rapid Geriatric Assessment: a tool for primary care physicians. J Am Medl Dir Assoc. 2017;18(3)191-9. Disponível em: https://doi.org/10.1016/j.jamda.2016.11.017.

3. Fonseca DC, Nascimento KCCG, Fialho FS, Lustosa MM, Dias RS. Prevalência de síndromes geriátricas em uma população de uma unidade de saúde do Pará. Rev Artigos.com. 2019;19:e4235. Disponível em: https://acervomais.com.br/index. php/artigos/article/view/4235 .

4. Fried LP, Tangen CM, Walston J, Newman AB, Hirsch C, Gottdiener J, et al. Frailty in older adults: evidence for a phenotype. J Gerontol Ser A Biol Sci Med Sci. 2001;56(3):146-56. Disponível em: https:// doi.org/10.1093/gerona/56.3.m146 .

5. Cesari M, Demougeot L, Boccalon H, Guyonnet S, Abellan Van Kan G, Vellas B, et al. A self-reported screening tool for detecting community-dwelling older persons with frailty syndrome in the absence of mobility disability: the FiND questionnaire. PLoS ONE. 2014;9(7):1-10. Disponível em: https://doi. org/10.1371/journal.pone.0101745 .

6. Lourenço RA, Moreira VG, Mello RGB, Santos IS, Lin SM, Pinto ALF, et al. Consenso brasileiro sobre fragilidade em idosos: conceitos, epidemiologia e instrumentos de avaliação. Geriatr Gerontol Aging. 2018;12(2):121-35. Disponível em: http://doi. org/10.5327/Z2447-211520181800023 .

7. Beaton DE, Bombardier C, Guillemin F, Ferraz MB. Guidelines for the process of crosscultural adaptation of self-report measures. Vol. 25, Spine. 2000. Disponível em: https://doi. org/10.1097/00007632-200012150-00014

8. Carvalho VA, Caramelli P. Brazilian adaptation of the Addenbrooke's Cognitive Examination-Revised (ACE-R). Dement Neuropsychol. 2007;1(2):1-10. Disponível em: https://doi.org/10.1590/S198057642008DN10200015.

9. Almeida OP, Almeida SA. Confiabilidade da versão brasileira da Escala de Depressão em Geriatria (GDS) versão reduzida. Arq Neuropsiquiatr. 1999;57(2B):110. Disponível em: https://doi.org/10.1590/S0004282X1999000300013.
10. Vellas B, Guigoz Y, Garry PJ, Nourhashemi F, Bennahum D, Lauque $S$, et al. The mini nutritional assessment (MNA) and its use in grading the nutritional state of elderly patients. Nutrition. 1999;15(2):1-10. Disponível em: https://doi.org/ 10.1016/s0899-9007(98)00171-3.

11. Ciconelli RM, Ferraz MB, Santos W, Meinão I, Quaresma MR. Tradução para a língua portuguesa e validação do questionário genérico de avaliação de qualidade de vida SF-36 (Brasil SF-36). Rev Bras Reumatol. 1999;39(3):143-50. Disponível em: https://www.scielo.br/j/csc/a/ PwJPp5MtSZvLWfnFvszrX8h/?format=pdf\&lang=pt .

12. Sürücü L, Maslakçi A. Validity and reliability in quantitative research. Bus Manag Stud An Int J. 2020;8(3)2694-2726. Disponível em: https:/doi.org/ https://doi.org/10.15295/bmij.v8i3.1540

13. de Souza AC, Alexandre NMC, Guirardello EB. Propriedades psicométricas na avaliação de instrumentos: avaliação da confiabilidade e da validade. Epidemiol Serv Saúde. 2017;26(3):1-10. Disponível em: https://doi.org/10.5123/S16794974201700030002214 .

14. Levin J, Fox JA. Estatistica para ciências humanas. $9^{\circ}$ ed. São Paulo: Pearson Prentce Hall; 2006.

15. Ying GS, Maguire MG, Glynn RJ, Rosner B. Calculating sensitivity, specificity, and predictive values for correlated eye data. Investig Ophthalmol Vis Sci. 2020;61(11):1-10. Disponível em: https:// dx.doi.org/10.1167/iovs.61.11.29

16. Ajzen I, Fishbein M. Understanding attitudes and predicting social behaviour. New Jersey: PrenticeHall; 1980.

17. Nora CRD, Zoboli E, Vieira MM. Validação por peritos: importância na tradução e adaptação de instrumentos. Rev Gaúch Enferm. 2018;38(3):110. Disponível em: https://doi.org/10.1590/19831447.2017.03.64851.

18. Mokkink LB, Prinsen CAC, Bouter LM, de Vet HCW, Terwee CB. The Consensus-based standards for the selection of health measurement INstruments (COSMIN) and how to select an outcome measurement instrument. Braz J Phys Ther. 2016;20(2):1-10. Disponível em: https://doi. org/10.1590/bjpt-rbf.2014.0143 .

19. Augusti ACV, Falsarella GR, Coimbra AMV. Análise da síndrome da fragilidade em idosos na atenção primária: Estudo transversal. Rev Bras Med Fam Comunidade. 2017;12(39):1-10. Disponível em: https://doi.org/10.5712/rbmfc12(39)1353 . 
20. Silveira EA, Vieira LL, de Souza JD. Elevada prevalência de obesidade abdominal em idosos e associação com diabetes, hipertensão e doenças respiratórias. Ciênc Saúde Colet. 2018;23(3):1-10. Disponível em: https://doi.org/10.1590/141381232018233.01612016 .

21. dos Santos IS, Toledo NN, Siqueira TM, de Oliveira APP. Perfil dos idosos no interior do Amazonas. Enferm Rev. 2018;21(2):1-10. Disponível em: http://periodicos.pucminas.br/index.php/ enfermagemrevista/article/view/18820 .

22. Vaingankar JA, Abdin E, Chong SA, Sambasivam $\mathrm{R}$, Jeyagurunathan A, Seow E, et al. Psychometric properties of the positive mental health instrument among people with mental disorders: a crosssectional study. Health Qual Life Outcomes. 2016;14(1):1-10. Disponível em: https://doi. org/10.1186/s12955-016-0424-8 .

23. Brigola AG, Rossetti ES, dos Santos BR, Neri AL, Zazzetta MS, Inouye K, et al. Relationship between cognition and frailty in elderly: a systematic review. Dement Neuropsychol. 2015;9(2):1-10. Disponível em: https://doi.org/10.1590/1980-57642015DN92000005 .

24. Lutomski JE, Baars MAE, Van Kempen JA, Buurman BM, Den Elzen WPJ, Jansen APD, et al. Validation of a frailty index from the older persons and informal caregivers survey minimum data set. J Am Geriatr Soc. 2013;61(9):1-10. Disponível em: https://doi. org/10.1111/jgs.12430 .

25. de Souza OF, Brochine LR, Gomes DJ, Novais MH, Zazzetta MS, Iost PSC, et al. Translation, adaptation and validation of rapid geriatric assessment to the Brazilian context. J Nutr Health Aging. 2018;22(9):1115-21. Disponível em: https://doi. org/10.1007/s12603-018-1078-5 .
26. Mirabelli LG, Cosker RM, Kraiss LW, Griffin CL, Smith BK, Sarfati MR, et al. Rapid methods for routine frailty assessment during vascular surgery clinic visits. Ann Vasc Surg. 2018;46:1-9. Disponível em: https://doi.org/10.1016/j.avsg.2017.08.010 .

27. Rossetti ES, Terassi M, Ottaviani AC, dos Santos OAA, Pavarini SCI, Zazzetta MS. Fragilidade, sintomas depressivos e sobrecarga de idosos cuidadores em contexto de alta vulnerabilidade social. Texto \& Contexto Enferm. 2018;27(3):1-9. Disponível em: https://doi.org/10.1590/010407072018003590016 .

28. Ribeiro O, Duarte N, Teixeira L, Paúl C. Frailty and depression in centenarians. Int Psychogeriatr. 2018;30(1):1-9. Disponível em: https://doi. org/10.1017/S1041610217001910 .

29. de Jesus ITM, Diniz MAA, Lanzotti RB, Orlandi FS, Pavarin SCI, Zazzetta MS. Fragilidade e qualidade de vida de idosos em contexto de vulnerabilidade social. Texto \& Contexto Enferm. 2018;27(4):1-9. Disponível em: https://doi.org/10.1590/010407072018004300016 .

30. Kojima G, Iliffe S, Jivraj S, Walters K. Association between frailty and quality of life among community-dwelling older people: a systematic review and meta-analysis. J Epidemiol Community Health. 2016;70:1-9. Disponível em: https:// doi.org/10.1136/jech-2015-206717 .

31. Jayanama K, Theou O, Blodgett JM, Cahill L, Rockwood K. Frailty, nutrition-related parameters, and mortality across the adult age spectrum. BMC Med. 2018;16(1):1-9. Disponível em: https://doi. org/10.1186/s12916-018-1176-6 .

32. Hao Q, Dong B, Yang M, Dong B, Wei Y. Frailty and cognitive impairment in predicting mortality among oldest-old people. Front Aging Neurosci. 2018;10:1-9. Disponível em: https://doi.org/10.3389/ fnagi.2018.00295 